\title{
Space-time supersymmetry, IIA/B duality and M-theory
}

\author{
Mohab Abou-Zeid ${ }^{\mathrm{a}, 1}$, Bernard de Wit ${ }^{\mathrm{a}, \mathrm{b}, 2}$, Dieter Lüst ${ }^{\mathrm{c}, 3}$, Hermann Nicolai ${ }^{\text {a,4 }}$ \\ ${ }^{a}$ Max Planck Institut für Gravitationsphysik, Albert Einstein Institut, Am Mühlenberg 5, D-14476 Golm, Germany \\ b Institute for Theoretical Physics, Utrecht University, Princetonplein 5, 3508 TA Utrecht, The Netherlands \\ ${ }^{\mathrm{c}}$ Institut für Physik, Humboldt Universität zu Berlin, Invalidenstrasse, D-10115 Berlin, Germany
}

Received 30 August 1999; accepted 23 September 1999

Editor: L. Alvarez-Gaumé

\begin{abstract}
The connection between IIA superstring theory compactified on a circle of radius $R$ and IIB theory compactified on a circle of radius $1 / R$ is reexamined from the perspective of $N=2, D=9$ space-time supersymmetry. We argue that the consistency of IIA/B duality requires the BPS states corresponding to momentum and winding of either of the type-II superstrings to transform as inequivalent supermultiplets. We show that this is indeed the case for any finite compactification radius, thus providing a nontrivial confirmation of IIA/B duality. From the point of view of $N=2, D=9$ supergravity, one is naturally led to an $\mathrm{SL}(2, \mathbb{Z})$ invariant field theory that encompasses both the M-theory torus and the Kaluza-Klein states of the IIB theory. (C) 1999 Published by Elsevier Science B.V. All rights reserved.
\end{abstract}

\section{Introduction}

The bosonic string compactified on a circle of radius $R$ is subject to a duality which relates the theory obtained at a compactification radius $R$ to the theory compactified at radius $1 / R$, where we set the string scale to unity [1]. The origin of this duality is that momentum modes, whose masses are multiples of $1 / R$ are accompanied by winding modes, whose masses are multiples of $R$. The spectrum exhibits a symmetry under $R \rightarrow 1 / R$ combined with an interchange of momentum and winding states, which in fact is a symmetry of the full string theory. Because

\footnotetext{
${ }^{1}$ E-mail: abouzeid@aei-potsdam.mpg.de

${ }^{2}$ E-mail: bdewit@phys.uu.nl

${ }^{3}$ E-mail: luest@physik.hu-berlin.de

${ }^{4}$ E-mail: nicolai@aei-potsdam.mpg.de
}

large and small distances are related, $R$ can be restricted to the interval $[1, \infty)$ and the theory appears to have a smallest length set by the string scale. At $R=\infty$ the theory decompactifies while at the self-dual point $R=1$ the winding and momentum states acquire equal masses and gauge symmetry enhancement takes place. The heterotic string possesses the same kind of duality symmetry [2].

However, there are situations where string theory is not self-dual in this naive sense, although the spectra at compactification scales $R$ and $1 / R$ seem to be identical. This is the case for the type-II string theories [3,4]. One way to analyze whether or not the theory is self-dual is to start at large compactification radius and to extrapolate all the way to zero radius. In that limit, the winding states become massless and the theory is expected to again decompactify. If this is indeed the case, one must obtain one of the consistent string theories defined in the uncompacti- 
fied space-time; either this is the theory one started with, or it is a different string theory.

The approach followed in this paper is that one can understand which theories are related by duality without extrapolating to zero radius provided the winding and momentum states carry different spacetime quantum numbers from the very beginning, where by space-time we refer to the generic spacetime with one compactified coordinate of finite radius. We claim that this happens to the type-II string theories, where we will show that the momentum and winding states constitute inequivalent $N=2$, $D=9$ supermultiplets at any given compactification radius. In this situation two immediate conclusions are obvious. First of all, there will be no symmetry enhancement when the masses of momentum and winding states become equal (this is consistent with the fact that the underlying conformal theory does not give rise to gauge symmetry enhancement), and secondly, the uncompactified theory obtained at $R=$ 0 is distinct from the theory at $R=\infty$. In this case, duality is conceptually different from a symmetry. Clearly one must find a different theory for any value of the compactification radius. There are no two radii at which the corresponding theories could conceivably be identical, because their spectra will be inequivalent; thus the different theories are now parametrized by the radius $R$ in the interval $(0, \infty)$. Duality means that two theories that are unrelated in the uncompactified space-time, can be viewed as different limits in the 'moduli' space of the compactified theories. Of course, one can describe the compactified theory from the perspective of one of the two inequivalent uncompacified theories associated with the endpoints $R=\infty$ and $R=0$, but this leaves it unchanged.

The present evidence for IIA/B duality is either based on formally mapping one theory onto the other, or on studying the behaviour near the two decompactification points at $R=\infty$ and $R=0$. In [3] it was argued that the consistency of the interchange of momentum and winding numbers, and of $R$ and $1 / R$, with world-sheet superconformal invariance requires that the components in the compactified direction of both the left-moving bosonic and fermionic world-sheet fields change sign, leading to a corresponding flip in the GSO projection [5]. Formally, one thus obtains a mapping between two consistent theories, namely compactified IIA and compactified IIB string theory, which extends to their respective vertex operators. In [4] a possible continuous connection between the two theories is investigated by considering the Lorentz generators near the two decompactification points. The sign change of the world-sheet fields is invoked in order to show that the Lorentz representations carried by the Ramond-Ramond ground state in these two limits must be different. In this approach, however (as noted in [4]), the ten-dimensional Lorentz invariance is broken when the compactification radius $R$ is different from zero or infinity, so that one cannot truly interpolate between the two theories.

The arguments given in this paper provide additional evidence that the IIA and the IIB theories are asymptotic limits in a one-parameter moduli space of theories. First, we analyze the nine-dimensional $N=$ 2 supersymmetry algebra from the space-time viewpoint and on the basis of perturbative string theory and M-theory. This analysis indeed confirms that the winding and momentum states constitute inequivalent supermultiplets in either of the type-II string theories at a given compactification radius. We then show that the same conclusion follows upon imposing the physical state conditions on the relevant string vertex operators. In fact, $N=2, D=9$ supersymmetry alone already gives rise to a unified description encompassing both the M-theory torus and the Kaluza-Klein states of the IIB theory. Our work lends support to the arguments given in [6] that there is a duality between M-theory and IIB theory which can be understood in terms of the fundamental supermembrane [7]. The coupling of one class of BPS states to supergravity breaks the continuous $\operatorname{SL}(2, \mathbb{R})$ symmetry group into a discrete subgroup associated with the Kaluza-Klein states on $T^{2}$. The second class of BPS multiplets carries charges that are unrelated to $T^{2}$ and can be identified with either the KaluzaKlein states of the IIB theory, or the wrapping of a membrane around the torus.

\section{2. $N=2$ supersymmetry in nine dimensions}

Let us first summarize the various possible BPS multiplets associated with the nine-dimensional supersymmetry algebra with Lorentz invariant central 
charges. We consider the supersymmetry algebra in an $N=2$ Majorana basis and write it as follows,

$$
\left\{Q_{\alpha}^{i}, Q_{\beta}^{\dagger j}\right\}=\left(P \gamma^{0}\right)_{\alpha \beta}+Z^{i j}\left(i \gamma^{0}\right)_{\alpha \beta} \text {. }
$$

We recall that, in nine dimensions, the charge conjugation matrix is symmetric and can be chosen equal to the identity; therefore $Z^{i j}$ is a real symmetric matrix. We can decompose the central charge as

$Z^{i j}=M\left[b \delta^{i j}+a\left(\cos \theta \sigma_{3}+\sin \theta \sigma_{1}\right)^{i j}\right]$,

where $M$ is the rest mass of the representation and $\sigma_{1}, \sigma_{2}, \sigma_{3}$ denote the usual Pauli matrices. It is clear, and this is crucial for what follows, that these central charges fall into two categories. The component proportional to $b$ defines an $\mathrm{SO}(2)$ invariant central charge, while the two components proportional to $a$ rotate into each other under the action of the automorphism group $\mathrm{SO}(2)$.

Let us first derive the possible values for $Z^{i j}$ when straightforwardly reducing the ten-dimensional supersymmetry algebra. We first decompose the Clifford algebra generated by the ten-dimensional gamma matrices $\Gamma^{M}$, and define $\Gamma^{11}$ by $\Gamma^{11}=$ $\Gamma^{0} \Gamma^{1} \cdots \Gamma^{9}$. Nine-dimensional gamma matrices, which commute with $\Gamma^{9}$ and $\Gamma^{11}$, are given by

$\gamma^{\mu}=\Gamma^{\mu} \tilde{\gamma}, \quad \mu=0,1,2, \ldots, 8$,

where $\tilde{\gamma}=-i \Gamma_{9} \Gamma_{11}$, such that the product $\gamma^{0} \gamma^{1}$ $\cdots \gamma^{8}=-i \mathbf{1}$. Observe that the Dirac conjugate of a spinor is changed accordingly ${ }^{5}$. The ten-dimensional charge-conjugation matrix can now be written as $\tilde{\gamma}$, so that the nine-dimensional gamma matrices are symmetric and the nine-dimensional charge-conjugation matrix equals the unit matrix. With these conventions, the ten-dimensional supersymmetry algebra $\{Q, \bar{Q}\}=-i P_{M} \Gamma^{M}$ is converted into the nine-dimensional algebra $\{Q, \bar{Q}\}=-i P_{\mu} \gamma^{\mu}-$ $P_{9} \Gamma^{11}$. Hence the sign of the $P_{9}$ term depends on the chirality of the supercharge. Therefore, the matrix $Z^{i j}$ will be proportional to $\left(\sigma_{3}\right)^{i j}$ for IIA theory, where one has supercharges of opposite chirality,

\footnotetext{
${ }^{5}$ In nine and ten dimensions, the Dirac conjugate is defined by $\bar{\psi}=i \psi^{\dagger} \gamma^{0}$ and $\bar{\psi}=i \psi^{\dagger} \Gamma^{0}$, respectively. Note that the $\gamma^{\mu}$ and $\Gamma^{M}$ are hermitean, with the exception of $\gamma^{0}$ and $\Gamma^{0}$, which are anti-hermitean. We use the 'mostly plus' metric. Observe that our conventions are such that there is no $\Gamma^{10}$ matrix.
}

and proportional to $\delta^{i j}$ for IIB theory, where the charges have equal chirality.

To exhibit the BPS multiplets in nine dimensions we diagonalize the matrix (2) by an appropriate $\mathrm{SO}(2)$ transformation so that $\theta=0$. In the rest frame the anticommutator (1) decomposes into four eightdimensional unit matrices, according to the decomposition $\mathbf{8}_{c}+\mathbf{8}_{s}+\mathbf{8}_{c}+\mathbf{8}_{s}$ of the thirty-two supercharges, with coefficients equal to $M$ times $(1+a+$ $b),(1-a-b),(1-a+b)$ and $(1+a-b)$, respectively. We have BPS multiplets whenever one of these coefficients vanishes. So we distinguish the following three cases:

- Choosing $a= \pm 1$ and $b=0$ leads to the $\left(\mathbf{8}_{v}+\right.$ $\left.\mathbf{8}_{s}\right) \times\left(\mathbf{8}_{v}+\mathbf{8}_{c}\right)$ decomposition of the $2^{8}$-dimensional supermultiplet with respect to the rest-frame spin rotation group $\mathrm{SO}(8)$. As always we can combine multiplets into larger multiplets with higher spin (i.e. by assigning spin to the Clifford vacuum), but here we concentrate on the smallest multiplet. Note that this multiplet contains fermions of mixed chirality. Another characteristic feature is the presence of a $\mathbf{5 6}_{v}$ spin representation. This is the multiplet that comprises the Kaluza-Klein states of IIA supergravity compactified on $S^{1}$, which are the momentum states of the compactified IIA string. Therefore this particular multiplet will be called the KKA multiplet.

- Choosing $a=0$ and $b= \pm 1$ leads to the $2^{8}$-dimensional multiplet $\left(\mathbf{8}_{v}+\overline{8}_{c}\right) \times\left(\mathbf{8}_{v}+\mathbf{8}_{c}\right)$. Again we can obtain larger multiplets of higher spin, but these will not be discussed here. A sign change in $b$ leads to the conjugate multiplet, where $\mathbf{8}_{s}$ and $\mathbf{8}_{c}$ are interchanged. Obviously the fermions have definite chirality and their partners in the conjugate supermultiplet carry opposite chirality. Observe also the absence of $\mathbf{5 6}_{v}$ states. This supermultiplet comprises the momentum states of the IIB theory and therefore it will be called the KKB multiplet. Clearly, the BPS states associated with a membrane wrapped around $T^{2}$ in eleven dimensions constitute KKB multiplets. Observe that this is crucial for the duality between M-theory and IIB theory, noted in [6].

- The multiplets with $\pm a \pm b= \pm 1$ comprise $2^{12}$ states. They do appear in string theory as mixed states containing both winding and momentum and have a nonzero oscillator number in order to 
satisfy the mass-shell condition. Hence they carry masses of the order of the string scale. The smallest multiplet associated with the lowest spins decomposes as $\left(\mathbf{8}_{v}+\mathbf{8}_{c}\right) \times\left(\mathbf{8}_{v}+\mathbf{8}_{c}\right) \times\left(\mathbf{8}_{v}+\mathbf{8}_{s}\right)$. Again there is a conjugate multiplet when changing the signs of $a$ and $b$. This class of BPS supermultiplets will not play a role in what follows.

In the literature one often finds the statement that the IIA and the IIB theories become indistinguishable when viewed in a nine-dimensional context, because the $\mathrm{SO}(7)$ decompositions of the IIA and IIB massless multiplets coincide. Although this is true, it is essential to understand that the Kaluza-Klein momentum states for the two theories remain different in nine dimensions: for massive states in nine dimensions, the rest-frame $\mathrm{SO}(8)$ rotation group coincides with the $\mathrm{SO}(8)$ helicity group for massless states in ten dimensions.

It is furthermore important that the KKA and KKB multiplets differ not only in their spin decomposition, but also carry inequivalent charges. We will return to this shortly, but we already note here that a KKA supermultiplet carries a nonzero $\mathrm{SO}(2)$ doublet charge while a KKB supermultiplet carries the $\mathrm{SO}(2)$ invariant charge. It follows from the above observations that these charges are mutually exclusive for these multiplets (but not for the intermediate' multiplets with $2^{12}$ states). As explained in the introduction, it is of vital importance for duality between the two type-II string theories that the winding and the momentum modes of a given type-II theory at a given compactification radius constitute inequivalent representations and correspond to different kinds of string states.

The above conclusions can also be arrived at by consideration of the supersymmetry algebra in eleven dimensions with a membrane charge,

$\{Q, \bar{Q}\}=-i P_{\hat{M}} \Gamma^{\hat{M}}+\frac{1}{2} i Z_{\hat{M} \hat{N}} \Gamma^{\hat{M} \hat{N}}$,

where we have eleven-dimensional momenta $P_{\hat{M}}$, two-brane charges $Z_{\hat{M} \hat{N}}$ and 32-component spinor charges. Upon reducing this algebra to nine dimensions, assuming that $Z_{\hat{M} \hat{N}}$ takes only values in the two extra dimensions labeled by $\hat{M}=9,10$, we obtain for the central-charge matrix $Z^{i j}$,

$Z^{i j}=Z_{910} \delta^{i j}-\left(P_{9} \sigma_{3}-P_{10} \sigma_{1}\right)^{i j}$.
From this result, we deduce the general BPS mass formula,

$M=\sqrt{P_{9}^{2}+P_{10}^{2}}+\left|Z_{910}\right|$.

We will further elaborate on the significance of these formulas later.

\section{World sheet description}

The structure of the BPS supermultiplets can also be established on the basis of the world-sheet superconformal field theory. In type-II string theory, the two Majorana supercharges can be represented as contour integrals over world-sheet operators. One charge, $Q_{\alpha}^{1}$, resides in the left-moving sector and the other one, $Q_{\alpha}^{2}$, resides in the right-moving sector, so we define

$Q_{\alpha}^{1}=\oint \frac{d z}{2 \pi i} V_{\alpha}(z), \quad Q_{\alpha}^{2}=\oint \frac{d \bar{z}}{2 \pi i} V_{\alpha}(\bar{z})$.

In the canonical $q=-1 / 2$ ghost picture the two covariant left- and right-moving fermion vertex operators $V_{\alpha}(z)$ and $V_{\alpha}(\bar{z})$ are given by [8] (omitting normal-ordering symbols)

$$
\begin{aligned}
& V_{\alpha(-1 / 2)}(z)=\left(\alpha^{\prime}\right)^{-1 / 4} S_{\alpha}(z) \exp \left(-\frac{1}{2} \phi(z)\right), \\
& V_{\alpha(-1 / 2)}(\bar{z})=\left(\alpha^{\prime}\right)^{-1 / 4} S_{\alpha}(\bar{z}) \exp \left(-\frac{1}{2} \phi(\bar{z})\right),
\end{aligned}
$$

where $\phi(z)(\phi(\bar{z}))$ is one of the left- (right)-moving bosonized superconformal ghosts and $S_{\alpha}(z), S_{\alpha}(\bar{z})$ are the spin field vertex operators in the $\mathbf{1 6}$ or $\overline{\mathbf{1 6}}$ chiral spinor representations of $S O(9,1)$. Note that (8) is valid for both IIA and IIB string theory, as we refrain from using dotted and undotted indices to indicate the chirality. Whenever this may lead to confusion, the reader should remember to simply project onto the corresponding chiral subspaces. The ten world-sheet fermions $\psi^{M}(z)$ can be bosonized in terms of five scalars $\boldsymbol{\phi}$ as $\exp \left(i \boldsymbol{\lambda}_{\mathrm{v}} \cdot \boldsymbol{\phi}\right)$, with the $\mathrm{SO}(1,9)$ vector weights $\boldsymbol{\lambda}_{\mathrm{v}}=(0, \ldots, \pm 1,0, \ldots)$ (thus $\left.\lambda_{\mathrm{v}}^{2}=1\right)$. The spin field operators $S_{\alpha}(z)$ can be similarly expressed as $\exp \left(i \boldsymbol{\lambda}_{\mathrm{s}} \cdot \boldsymbol{\phi}(z)\right)$, or $\exp \left(i \boldsymbol{\lambda}_{\mathrm{c}}\right.$. $\boldsymbol{\phi}(z))$, where $\boldsymbol{\lambda}_{\mathrm{s}}$ and $\boldsymbol{\lambda}_{\mathrm{c}}$ denote the two $\operatorname{SO}(9,1)$ chiral spinor weights $\left( \pm \frac{1}{2}, \pm \frac{1}{2}, \pm \frac{1}{2}, \pm \frac{1}{2}, \pm \frac{1}{2}\right)$, with 
an even (odd) number of minus signs for the positive (negative) chirality (and $\lambda_{\mathrm{s}}^{2}=\lambda_{\mathrm{c}}^{2}=\frac{5}{4}$ ).

In order to compute the supersymmetry algebra from these world sheet fields it is convenient to introduce the supercharges in the equivalent $q=$ $+1 / 2$ superconformal ghost picture. In this ghost picture $V_{\alpha}(z)$, for example, takes the form

$$
\begin{aligned}
V_{\alpha(+1 / 2)}(z)= & \left(\alpha^{\prime}\right)^{-3 / 4} \partial X_{L}^{M}(z)\left(\Gamma_{M} S(z)\right)_{\alpha} \\
& \times \exp \left(\frac{1}{2} \phi(z)\right),
\end{aligned}
$$

where the chirality of $S_{\alpha}$ is opposite to the chirality of the $S_{\alpha}$ used in the corresponding expression (8). The supersymmetry algebra can now be obtained by computing the following operator products between the vertex operators in the two different ghost pictures,

$$
\begin{aligned}
& V_{\alpha(-1 / 2)}(z) V_{\beta(+1 / 2)}(w) \\
& \quad \sim \frac{1}{z-w} \frac{1}{\alpha^{\prime}}\left(C \Gamma_{M}\right)_{\alpha \beta} \partial X_{L}^{M}(w)+\cdots, \\
& V_{\alpha(-1 / 2)}(\bar{z}) V_{\beta(+1 / 2)}(\bar{w}) \\
& \quad \sim \frac{1}{\bar{z}-\bar{w}} \frac{1}{\alpha^{\prime}}\left(C \Gamma_{M}\right)_{\alpha \beta} \bar{\partial} X_{R}^{M}(\bar{w})+\cdots, \\
& V_{\alpha(-1 / 2)}(z) V_{\beta(+1 / 2)}(\bar{w}) \sim 0,
\end{aligned}
$$

where $C$ is the ten-dimensional charge-conjugation matrix. Taking the contour integrals and converting to the nine-dimensional gamma indices introduced earlier, we find the $\left\{Q^{i}, \bar{Q}^{j}\right\}$ anticommutator in terms of the nine-dimensional momenta and the right- and left-moving zero-mode momenta,

$$
\begin{aligned}
& p_{L}=\frac{1}{\alpha^{\prime}} \oint \frac{d z}{2 \pi i} i \partial X_{L}^{9}(z)=\frac{1}{\sqrt{\alpha^{\prime}}}\left(\frac{m}{T}-n T\right), \\
& p_{R}=\frac{1}{\alpha^{\prime}} \oint \frac{d \bar{z}}{2 \pi i} i \bar{\partial} X_{R}^{9}(\bar{z})=\frac{1}{\sqrt{\alpha^{\prime}}}\left(\frac{m}{T}+n T\right),
\end{aligned}
$$

where we measure the compactification radius $R$ in string units by means of a dimensionless parameter $T=R / \sqrt{\alpha^{\prime}}$. The integers $m$ and $n$ denote the momentum and winding numbers, respectively. This yields the following supersymmetry algebra for the IIA/B superstrings in nine space-time dimensions

$$
\begin{aligned}
& \left\{Q^{1}, \bar{Q}^{1}\right\}=-i P_{\mu} \gamma^{\mu}-p_{L} \Gamma^{11}, \\
& \left\{Q^{2}, \bar{Q}^{2}\right\}=-i P_{\mu} \gamma^{\mu}-p_{R} \Gamma^{11}, \\
& \left\{Q^{1}, \bar{Q}^{2}\right\}=0 .
\end{aligned}
$$

Comparing with the previously derived supersymmetry algebra (1), it is now obvious that the central charges are just linear combinations of the internal left- and right momenta $p_{L}$ and $p_{R}$. To be more precise, in the IIA and the IIB theory the central charge matrix $Z^{i j}$ takes one of the two alternative forms (up to an overall sign),

$Z^{i j}= \begin{cases}\frac{1}{2}\left(p_{L}+p_{R}\right) \delta^{i j}+\frac{1}{2}\left(p_{L}-p_{R}\right)\left(\sigma_{3}\right)^{i j} & \text { for IIB } \\ \frac{1}{2}\left(p_{L}-p_{R}\right) \delta^{i j}+\frac{1}{2}\left(p_{L}+p_{R}\right)\left(\sigma_{3}\right)^{i j} & \text { for IIA }\end{cases}$

This proves our assertion that the momentum and winding BPS states constitute inequivalent supermultiplets. The IIA momentum states and the IIB winding states are in the KKA representation, whereas the IIA winding states and the IIB momentum states are in the KKB representation. This ensures that the two decompactification limits $T \rightarrow 0$ and $T \rightarrow \infty$ lead to different theories. Moreover, it proves that type-II string compactifications on circles of different radii must be inequivalent. And finally, it is clear that no symmetry enhancement will take place when the momentum and the winding states have coinciding masses, as these states are always distinctly different. This is in accord with the fact that no gauge symmetry enhancement is possible in the conformal field theory.

The emergence of different representations for the momentum and winding states can also be understood in terms of the corresponding covariant physical vertex operators. To write them down for the compactified theory in nine dimensions, we again make use of the $\mathrm{SO}(1,9)$ covariant ghost and spin field vertex operators. The vertex operators for the Kaluza-Klein and winding states in nine dimensions can be directly obtained from the vertex operators of the massless states in ten dimensions by splitting the 
physical momenta as in (12). More precisely, we consider the Ramond-Ramond operators

$$
\begin{aligned}
& \exp \left(i p_{\mu} X_{L}^{\mu}(z)+i p_{L} X_{L}^{9}(z)\right) \bar{u}_{L}^{\alpha}(p) S_{\alpha}(z) \\
& \quad \times \exp \left(-\frac{1}{2} \phi(z)\right) \exp \left(i p_{\mu} X_{R}^{\mu}(\bar{z})+i p_{R} X_{R}^{9}(\bar{z})\right) \\
& \quad \times \bar{u}_{R}^{\beta}(p) S_{\beta}(\bar{z}) \exp \left(-\frac{1}{2} \phi(\bar{z})\right)
\end{aligned}
$$

where the 16-component spinors $u_{L}(p)$ and $u_{R}(p)$ denote the chiral $\mathrm{SO}(1,9)$ spinor polarizations of the left- and right-moving states (so that we have implemented a GSO projection) and the $p_{\mu}$ are the values taken by the nine-dimensional momentum operators $P_{\mu}$. Again we refrain from using dotted and undotted $\mathrm{SO}(1,9)$ spinor indices and we leave the chirality of the spin fields and therefore of the polarization spinors unspecified. Note, however, that the chirality of the $S_{\alpha}$ must be the same as in (8). We recall also that the ghost and spinor weights for the vertex operator must be chosen in accordance with the locality requirement (see [9] for a detailed discussion of this point).

To the operators (14) we must apply the physical state condition which follows from requiring that they commute with the left- and right-moving BRST operators. The relevant part of the left-moving such operator is proportional to $\partial X_{L}^{M}(z) \psi_{L M}(z) \times$ $\exp (\phi(z))$; the formula for its right-moving counterpart is similar. In this way we recover first of all the mass-shell condition $-p_{\mu} p^{\mu}=p_{L}^{2}=p_{R}^{2}$, where the last equality is valid only for states without oscillator excitations. Secondly, we obtain the Dirac equation for the spinor polarizations. Written with nine-dimensional gamma matrices, this yields,

$$
\begin{aligned}
& \left(i p_{\mu} \gamma^{\mu}+p_{L} \Gamma^{11}\right) u_{L}(p) \\
& \quad=\left(i p_{\mu} \gamma^{\mu}+p_{R} \Gamma^{11}\right) u_{R}(p)=0 .
\end{aligned}
$$

These conditions reduce the number of physical spinor polarizations from 16 to 8 , so that the vertex operators (14) describe $8 \times 8=64$ states for given momentum. When combined with the NeveuSchwarz sector these states comprise full BPS supermultiplets. In obtaining the $\mathrm{SO}(8)$ representations in accord with our earlier analysis, it is important to realize that the chirality of the polarization spinors is opposite to that of the corresponding $S_{\alpha}$.

The above results are in precise correspondence with our previous analysis of the superalgebra relations (12). The mass-shell condition tells us that $p_{L}= \pm p_{R}$, and depending on this sign, we get either the same or different $\mathrm{SO}(8)$ representations from the physical state condition (15). Thus winding and momentum states indeed constitute inequivalent supermultiplets.

Finally let us discuss the choice of the chirality for the spinors in (8) and in (14). Clearly we only need to distinguish between equal and opposite chirality for the left- and right-moving spinor fields. On the other hand, switching the relative chirality, e.g. by changing the chirality of $S_{\alpha}(z)$, and correspondingly of $u_{L}$, can be compensated for by assigning an opposite momentum $p_{L}$ to that state, leaving $p_{R}$ unchanged. This corresponds to interchanging the winding and the momentum numbers $m$ and $n$ in (11), together with the interchange of $T$ with $1 / T$. So the states and the corresponding supermultiplets remain the same; what changes is only the notion of a momentum and a winding state. Clearly, there is a type-IIA and a type-IIB description, but of a single theory. In the decompactification limits $T \rightarrow 0$ and $T \rightarrow \infty$, one is left with inequivalent supermultiplets as the mass of one supermultiplet vanishes and that of its inequivalent counterpart is pushed to infinity.

It is equally straightforward to analyze the 'intermediate' BPS multiplets with $2^{12}$ states from this point of view. However, the corresponding vertex operators are more complicated due to oscillator contributions, which modifies the relation between $p_{L}$ and $p_{R}$.

\section{4. $N=2$ supergravity in nine dimensions}

We will now use $N=2$ supergravity in $D=9$ dimensions together with some basic input from string theory to obtain independent confirmation of the result that the momentum and winding states are in different supermultiplets. Let us first discuss some features of the massless fields which constitute $N=2$ supergravity in nine dimensions. This theory has already been discussed in the literature; in particular, its relation to string theory and IIA/B duality was 
studied in [10], so we will be brief here. The focus of our attention is the coupling of the massless theory to the massive BPS states that we discussed above. In particular, we want to exhibit the coupling of the nine-dimensional gauge fields to the BPS states.

In nine dimensions there is only one $N=2$ supergravity theory, whose scalar sector is governed by an $\mathrm{SL}(2, \mathbb{R}) / \mathrm{SO}(2)$ non-linear $\sigma$-model, and which therefore exhibits an invariance under a nonlinearly realized $\operatorname{SL}(2, \mathbb{R})$. In addition there is an invariance under $\mathrm{SO}(1,1)$, which can be systematically understood from combining ordinary dimensional analysis with scale transformations on the compactified coordinate [11]. From the IIB supergravity perspective, the $\operatorname{SL}(2, \mathbb{R})$ originates from the $\operatorname{SL}(2, \mathbb{R}) / \mathrm{SO}(2)$ coset structure and the $\operatorname{SL}(2, \mathbb{R})$ symmetry which are already present in ten dimensions [12]. From the perspective of eleven-dimensional supergravity [13], on the other hand, these are just the 'hidden' symmetries obtained by reducing the theory from eleven to nine dimensions on the torus $T^{2}$. In this reduction, the diffeomorphism symmetry in the compactified dimensions is 'frozen' to a rigid $\operatorname{GL}(2, \mathbb{R})=\operatorname{SL}(2, \mathbb{R})$ $\times \operatorname{SO}(1,1)$ symmetry. Similarly, the full Lorentz symmetry in eleven dimensions is reduced to $\mathrm{SO}(1,8) \times \mathrm{SO}(2) \subset \mathrm{SO}(1,10)$, where $\mathrm{SO}(2)$ is converted into the R-symmetry corresponding to the automorphism group of the nine-dimensional $N=2$ superalgebra.

Identifying the various transformations, one readily obtains the various quantum numbers, without the need for a detailed dimensional reduction. We denote the bosonic fields of eleven-dimensional supergravity by $\hat{G}_{\hat{M} \hat{N}}$ and $\hat{A_{\hat{M} \hat{N}}}$. The bosonic fields of IIA supergravity are denoted by $G_{M N}, C_{M}, C_{M N}, C_{M N P}$ and $\phi$, and those of IIB supergravity by $G_{M N}, A_{M N}^{\alpha}$, $\phi^{\alpha}$ and $A_{M N P Q}$. Here the index $\alpha$ is associated with $\mathrm{SL}(2, \mathbb{R})$. The fields of $N=2$ nine-dimensional supergravity are the metric $g_{\mu \nu}$, three scalars $\sigma$ and $\phi^{\alpha}$, three abelian gauge fields $B_{\mu}$ and $A_{\mu}^{\alpha}$, two antisymmetric tensors $A_{\mu \nu}^{\alpha}$ and a three-rank antisymmetric gauge field $A_{\mu \nu \rho}$. The fields and their $\mathrm{SO}(1,1)$ weights are summarized in Table 1 . We use the Einstein frame, so that the metric is invariant under $\mathrm{SO}(1,1)$. The scalar fields $\phi^{\alpha}$ characterize the coset representative of $\operatorname{SL}(2, \mathbb{R}) / \mathrm{SO}(2)$. They satisfy a constraint $\phi^{\alpha} \phi_{\alpha}=1$ and are subject to local $\mathrm{SO}(2)$ transformations, so that they correspond to one com-
Table 1

The bosonic fields of the eleven dimensional, type-IIA, nine-dimensional $N=2$ and type-IIB supergravity theories. The elevendimensional and ten-dimensional indices, respectively, are split as $\hat{M}=(\mu, 9,10)$ and $M=(\mu, 9)$, where $\mu=0,1, \ldots 8$. The last column lists the $\mathrm{SO}(1,1)$ scaling weights of the fields.

\begin{tabular}{llllc}
\hline$D=11$ & IIA & $D=9$ & IIB & SO(1,1) \\
\hline$\hat{G}_{\mu \nu}$ & $G_{\mu \nu}$ & $g_{\mu \nu}$ & $G_{\mu \nu}$ & 0 \\
$\hat{A_{\mu 910}}$ & $C_{\mu 9}$ & $B_{\mu}$ & $G_{\mu 9}$ & -4 \\
$\hat{G}_{\mu 9}, \hat{G}_{\mu 10}$ & $G_{\mu 9}, C_{\mu}$ & $A_{\mu}^{\alpha}$ & $A_{\mu 9}^{\alpha}$ & 3 \\
$\hat{A_{\mu \nu 9}}, \hat{A_{\mu \nu} 10}$ & $C_{\mu \nu 9}, C_{\mu \nu}$ & $A_{\mu \nu}^{\alpha}$ & $A_{\mu \nu}^{\alpha}$ & -1 \\
$\hat{A_{\mu \nu \rho}}$ & $C_{\mu \nu \rho}$ & $A_{\mu \nu \rho}$ & $A_{\mu \nu \rho \sigma}$ & 2 \\
$\hat{G}_{910}, \hat{G}_{99}, \hat{G}_{1010}$ & $\phi, G_{99}, C_{9}$ & $\left\{\begin{array}{l}\phi^{\alpha} \\
\exp (\sigma)\end{array}\right.$ & $\phi_{99}^{\alpha}$ & 0 \\
\hline
\end{tabular}

plex field. The scalar $\exp (\sigma)$ will be defined as $G_{99}$, the IIB metric in the compactified dimension. The determinant of the eleven-dimensional metric in the two compactified directions is then equal to $\exp \left(-\frac{4}{3} \sigma\right)$. We have ignored certain nonlinear features of the relationship with the higher-dimensional fields. On the other hand, the assignments are also relevant for the massive Kaluza-Klein states in the $T^{2}$ and $S^{1}$ compactifications [11].

Now we consider the three abelian vector gauge fields in the nine-dimensional theory, which decompose into a singlet and a doublet under $\operatorname{SL}(2, \mathbb{R})$. Note that their origin is rather different when viewed from the IIA and from the IIB side. The singlet field is the graviphoton from the IIB side, so it must couple to the IIB momentum states. The doublet fields originate from the IIB doublet of tensor fields, so they couple to the IIB winding states. It thus follows that the IIB momentum states constitute KKB states (by definition) whereas the IIB winding states constitute KKA multiplets. The second KKA charge can only be understood beyond string perturbation theory; the degeneracy in the winding states is due to winding of fundamental and D-strings.

The pattern is the same, but complementary on the IIA side. Here the momentum states carry the doublet charges, so they constitute (again by definition) KKA multiplets. Accordingly, the two graviphotons originating from eleven dimensions transform as an $\operatorname{SL}(2, \mathbb{R})$ doublet. The degeneracy in the momentum states can thus be understood from 
eleven-dimensional supergravity, as the doublet charges find their origin in the $T^{2}$ on which the theory is compactified. The winding states couple to the singlet field, which originates from the IIA tensor field. Hence the IIA winding states constitute KKB multiplets. Alternatively these states can be understood as membranes wrapped around the M-theory torus [6], because, as we have shown before, these constitute the same supermultiplets.

\section{Coupling to BPS supermultiplets}

One may contemplate the construction of a ninedimensional field theory consisting of $N=2$ supergravity coupled to an infinite tower of BPS supermultiplets with a two-dimensional charge lattice $\left(q_{1}, q_{2}\right)$ for the KKA states and a one-dimensional lattice of charges $p$ for the KKB states. This theory encompasses both eleven-dimensional supergravity (compactified on $T^{2}$ ) and IIB supergravity (compactified on $S^{1}$ ). The usual T-duality is trivial for this theory. It is not associated with any symmetry and only amounts to certain field redefinitions. We know that the theory is free from inconsistencies in each of these sectors separately and it is an interesting question whether such a 'dichotomic' field theory could be (classically) consistent to all orders. In low orders of perturbation theory, its short-distance behaviour should be relatively mild as it can be viewed as a combination of known supergravity theories. Of course, this is not truly an effective field theory as the masses of the various states will never be light simultaneously with respect to the string scale. The theory is manifestly invariant under $\mathrm{SO}(1,1)$ and under $\operatorname{SL}(2, \mathbb{Z})$. The latter is the integer-valued subgroup of $\operatorname{SL}(2, \mathbb{R})$ that leaves the charge lattice of the KKA states invariant. There is a formulation in which the $\operatorname{SL}(2, \mathbb{R})$ is linearly realized, also in the presence of the BPS states. In that case the massive fields transform only under the local (composite) $\mathrm{SO}(2)$ and not directly under $\operatorname{SL}(2, \mathbb{R})$. However, the KKA fields have a minimal coupling with respect to $q_{\alpha} A_{\mu}^{\alpha}$, which, in order to remain invariant under the integer-valued subgroup, requires the charges to transform covariantly under this subgroup. The KKB fields have a minimal coupling to $p B_{\mu}$, which is $\operatorname{SL}(2, \mathbb{R})$ invariant.
It should be clear that the theory will exhibit tenor eleven-dimensional Lorentz invariance only in certain limits. For the KKA states with charges $q_{\alpha}$, and KKB states with charge $p$, respectively, the BPS mass formula in the nine-dimensional Einstein frame is given by

$M=m_{\mathrm{KKA}}\left|q_{\alpha} \phi^{\alpha}\right|+m_{\mathrm{KKB}}|p|$,

where $m_{\mathrm{KKA}}$ and $m_{\mathrm{KKB}}$ denote two different mass scales, whose product is inversely proportional to $\alpha^{\prime}$. Here we made use of the fact that the mass should be $\operatorname{SL}(2, \mathbb{Z})$ and $\mathrm{SO}(1,1)$ invariant in the Einstein frame.

As noted in [6] the mass formula (16) is entirely consistent with that of a membrane wrapped around a torus with modular parameter $\tau \equiv \tau_{1}+i \tau_{2}$. Here we should point out that the supersymmetry algebra for a fundamental supermembrane gives rise to precisely the algebra (4) with $Z_{\hat{M} \hat{N}}$ describing the winding of the membrane over some compact space [14]. In the case of a torus with area $A$, the BPS mass formula follows directly from (6) and reads (in eleven-dimensional Planck units)

$M=\frac{1}{\sqrt{A \tau_{2}}}\left|q_{1}-\tau q_{2}\right|+A T_{\mathrm{m}}|p|$,

where $T_{\mathrm{m}}$ denotes the supermembrane tension, $q_{1,2}$ label the momentum modes on the torus and $p$ is the number of times the membrane is wrapped (including orientation) over the torus. This formula agrees with the one previously derived in [15] on the basis of a semi-classical approximation. We refrain from indicating how the modular parameter is related to the fields $\phi^{\alpha}$ but simply note that both formulae are invariant under $\operatorname{SL}(2, \mathbb{Z})$.

The formula (16) can now be interpreted in two different ways. From the perspective of IIA string theory, one of the $q_{\alpha}$ is the IIA Kaluza-Klein momentum number, while the other is the D0 charge; as is well known, the mass of the D0 branes is inversely proportional to the IIA string coupling constant [16]. Then $p$ is the IIA winding number. Conversely, from the IIB perspective, $q_{1}$ and $q_{2}$ are the winding numbers of the elementary string and of the solitonic D1 string (which corresponds to a D0 brane in the IIA description). Now the $\operatorname{SL}(2, \mathbb{Z})$ is a strong-weak coupling duality, as it interchanges the elementary strings with the D1 strings. The modular parameter 
associated with the fields $\phi^{\alpha}$ is the IIB dilaton which contains the IIB string coupling constant. From this perspective the integral charge lattice follows from a Dirac-type quantization condition. The integer $p$ is just the IIB Kaluza-Klein momentum number.

The question that remains is, of course, what IIA/B duality can teach us about M-theory and its fundamental degrees of freedom. The theory we referred to as 'dichotomic' above transcends both eleven-dimensional supergravity and IIB supergravity. The above results can be interpreted as evidence that M-theory is just the fundamental supermembrane. Supermembrane theory may not suffer from the incompleteness of perturbative string theory. Unlike superstring theory, which has both a string tension as well as a coupling constant, it has no conventional perturbative expansion as its only parameter is the membrane tension $T_{\mathrm{m}}$. As is evident from (5) and (17), both the Kaluza-Klein doublet states and the winding states arise naturally upon compactification to nine dimensions. Likewise, the perturbative massive string states, which have no analog in the (uncompactified) supermembrane, can emerge out of the continuous supermembrane spectrum [17] in the reduction from eleven to ten dimensions (recall that the excited superstring states cannot be combined into massive $D=11$ multiplets). This indicates that the quantum supermembrane is not only a second quantized, but also a non-perturbative theory from the very outset - like M-theory.

\section{Acknowledgements}

We would like to thank Michael Green, Joseph Polchinski, Jorge Russo, Bert Schellekens and Nathan Seiberg for discussions. B.d.W. is grateful to the Alexander von Humboldt-Stiftung for supporting his stay at the AEI as part of the Humboldt Award Program. This work is supported in part by the European Commission TMR Program under contract ERBFMRX-CT96-0045, in which the Humboldt University at Berlin and Utrecht University are associated.

\section{References}

[1] K. Kikkawa and M. Yamasaki, Phys. Lett. B149 (1984) 357, N. Sakai and I. Senda, Prog. Theor. Phys. 75 (1986) 692; Erratum: ibid 77 (1987) 773.

[2] P. Ginsparg, Phys. Rev. D35 (1987) 648.

[3] M. Dine, P. Huet and N. Seiberg, Nucl. Phys. B322 (1989) 301.

[4] J. Dai, R.G. Leigh and J. Polchinski, Mod. Phys. Lett. A4 (1989) 2073.

[5] F. Gliozzi, J. Scherk and D. Olive, Phys. Lett. B65 (1976) 282; Nucl. Phys. B122 (1977) 253.

[6] J.H. Schwarz, Phys. Lett. B360 (1995) 13 [hep-th/9508143]; Erratum: ibid B364 (1995) 252; Nucl. Phys. Proc. Suppl. 49 (1996) 183 [hep-th/9509148]; Phys. Lett. B367 (1996) 97 [hep-th/9510086]; P.S. Aspinwall, Nucl. Phys. Proc. Suppl. 46 (1996) 30 [hep-th/9508154].

[7] E. Bergshoeff, E. Sezgin and P.K. Townsend, Phys. Lett. B189 (1987) 75.

[8] D. Friedan, E. Martinec and S. Shenker, Nucl. Phys. B271 (1986) 93.

[9] V. Kostelecký, O. Lechtenfeld, W. Lerche, S. Samuel and S. Watamura, Nucl. Phys. B288 (1987) 173.

[10] E. Bergshoeff, C. Hull and T. Ortin, Nucl. Phys. B451, 547 (1995) [hep-th/9504081].

[11] B. de Wit and J. Louis, Supersymmetry and Dualities in Various Dimensions [hep-th/9801132].

[12] J.H. Schwarz, Nucl. Phys. B226 (1983) 269.

[13] E. Cremmer, B. Julia and J. Scherk, Phys. Lett. B76 (1978) 409.

[14] B. de Wit, K. Peeters and J. Plefka, Phys. Lett. B409 (1997) 117 [hep-th/9705225].

[15] J. Russo and A. Tseytlin, Nucl. Phys. B490 (1997) 121 [hep-th /9611047].

[16] J. Polchinski, Phys. Rev. Lett. 75 (1995) 184 [hepth/9510017].

[17] B. de Wit, M. Lüscher and H. Nicolai, Nucl. Phys. B305 [FS23] (1990) 39. 